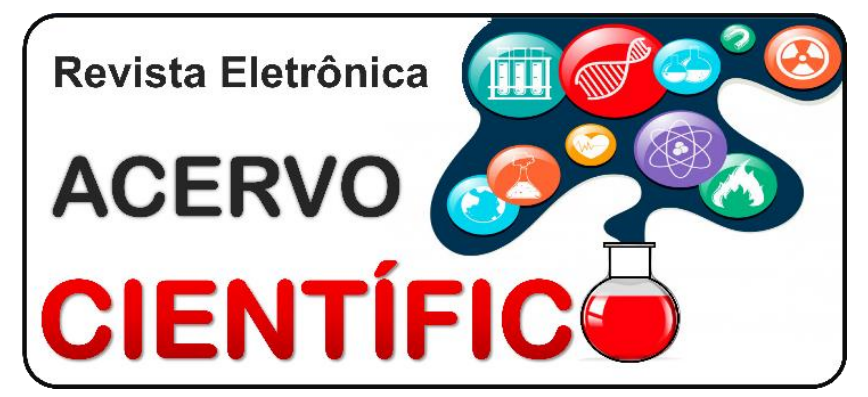

REVISÃO BIBLIOGRÁFICA

Recebido em: 6/2020

Aceito em: 7/2020

Publicado em: 12/2020

\title{
Avaliação das políticas públicas no contexto de portadores do HIV/AIDS: uma revisão integrativa da literatura
}

\author{
Evaluation of public policies in the context of HIV/AIDS patients: an integrative literature \\ review
}
Evaluación de políticas públicas en el contexto de pacientes con HIV/AIDS: una revisión integrativa de la literatura

Adilson Mendes de Figueiredo Júnior ${ }^{1 *}$, Milene de Nazaré Reis dos Santos ${ }^{1}$, Lorena Matos de Jesus $^{1}$, Lucas Monteiro Trindade ${ }^{1}$, Salvador Clebson Pureza Soares ${ }^{1}$, Tatheanne da Silva Trindade dos Santos ${ }^{1}$, Deisiane do Socorro da Silva Barbosa ${ }^{1}$, Giovanna Farias de Sousa ${ }^{2}$, Cristiane dos Santos Silva ${ }^{2}$, Edeiza Priscila de Souza Pinheiro ${ }^{3}$.

\begin{abstract}
Resumo: Este artigo buscou descrever as políticas públicas e sociais das pessoas vivendo com HIV/AIDS. Tratou-se de um estudo qualitativo e descritivo com método de revisão bibliográfica, que caracteriza-se como uma forma de revisão abrangente da literatura, e tem por objetivo fornecer evidências de investigação baseadas no tema "Políticas Públicas e Sociais para Pacientes com HIV/AIDS". Optou-se por organizar os resultados de acordo com o título do artigo, o estado de publicação, o ano de publicação e a base de dados onde foram descobertos. Foram respitadas todas as descobertas científicas e organizadas em uma tabela para melhor observação e compreenção de cada artigo científico. A avaliação epidemiológica do HIV/Aids permite observar como fatores (educação em saúde educação continuada e aspectos culturais) podem interferir no avanço de ações de promoção em saúde. Avaliar sua interferência deve estar intimamente associado a meios de evita-las, como: uso de preservativos, ambiente de trabalho e capacitação de equipes de saúde.
\end{abstract}

Palavras-chave: Políticas públicas, AIDS, Prevenção.

\begin{abstract}
This article sought to describe the public and social policies of people living with HIV / AIDS. It was a qualitative and descriptive study with a bibliographic review method, which is characterized as a form of comprehensive literature review, and aims to provide research evidence based on the theme "Public and Social Policies for Patients with HIV / AIDS". It was decided to organize the results according to the title of the article, the state of publication, the year of publication and the database where they were discovered. All scientific discoveries were respected and organized in a table for better observation and understanding. The epidemiological assessment of HIV / AIDS allows us to observe how factors (health education, continuing education and cultural aspects) can interfere in the advancement of health promotion actions. Assessing their interference must be closely associated with ways of avoiding them. , such as: condom use, work environment and training of health teams.
\end{abstract}

Keywords: Public policy, AIDS, Prevention.

1 Escola Superior da Amazônia (ESAMAZ), Belém - PA. *E-mail: adilsonmdfj@hotmail.com

2 Universidade do Estado do Pará (UEPA), Belém - PA.

${ }^{3}$ Centro Universitário do Pará (CESUPA), Belém - PA. 
Resumen: Este artículo buscó describir las políticas públicas y sociales de las personas que viven con el VIH / SIDA. Se trata de un estudio cualitativo y descriptivo con método de revisión bibliográfica, que se caracteriza por ser una forma de revisión bibliográfica integral, y tiene como objetivo aportar evidencia de investigación con base en el tema "Políticas Públicas y Sociales para Pacientes con VIH / SIDA". Se decidió organizar los resultados según el título del artículo, el estado de publicación, el año de publicación y la base de datos donde fueron descubiertos, todos los descubrimientos científicos fueron respetados y organizados en una tabla para una mejor observación y comprensión. La evaluación epidemiológica del VIH / SIDA permite observar cómo factores (educación para la salud, educación continua y aspectos culturales) pueden interferir en el avance de las acciones de promoción de la salud, valorar su interferencia debe estar estrechamente asociada a formas de evitarlas, tales como: uso del condón, ambiente laboral y capacitación de equipos de salud.

Palabras clave: Políticas públicas, AIDS, Prevención.

\section{INTRODUÇÃO}

A epidemia de HIV/AIDS ainda constitui um relevante problema de saúde pública no mundo e, principalmente no Brasil. Mesmo com tratamentos antirretrovirais e ações de educação em saúde no intuito de prevenção e promoção em saúde, não foi possível perceber a redução significante das taxas de incidência, de infectados e doentes. As estratégias mais presentes para a redução de contaminações e exposição aos riscos de adoecimento ainda são na sua grande parte direcionada ao tratamento de dados epidemiológicos que possam gerar resultados diretos em políticas públicas eficazes no âmbito universal (GUIMARÃES MDC, et al., 2017).

Levando em consideração a dinâmica que envolve a doença, a sociedade civil se mobilizou para organizar campanhas pelos direitos dos pacientes e redefinir o princípio do convívio social com as pessoas infectadas pelo vírus. A necessidade de se avaliar a estrutura político administrativa envolvida nesse contexto representa uma nova perspectiva de atuação profissional e até de respeito aos direitos dos portadores da síndrome ou apenas infectados pelo vírus (VILLARINHO MV e PADILHA MI, 2014).

As políticas públicas em saúde são utilizadas a partir de diversas fontes de dados de notificação de agravos e até mesmo um dado secundário dos modelos de vigilância, assim é possível relacionar tais dados com modelos estatísticos teóricos, o que permite a reclassificação das causas básicas de óbitos e, potencialmente, uma visão mais ampla da situação de mortalidade de uma determinada região ou país e, assim é possível entender diretamente o processo saúde $x$ doença que envolve $o$ agravo e agir diretamente sobre os meios de prevenção (GUIMARÃES MDC, et al., 2017).

As epidemias evidenciam um comportamento coletivo e são caracterizadas por uma associação multifatorial, não acontecem aleatoriamente, sendo ainda produto da combinação sociodemográfica, culturais, decisões políticas e do comportamento social de cada grupo social em determinado tempo. Assim, surge a necessidade de entender que o pensamento preventivo do HIV/AIDS não representa um pensamento único e isolado, mas um contexto social, político e ideológico complexo que sofre influência do tempo e necessita ser estudada frequentemente para um cuidado efetivo e uma atenção em saúde satisfatória (KADRI M e SCHWEICKARDT JC, 2016).

A paritr do apresentado, e com o intuito de fortalecer o reconhecimento e enfatizar o debate em torno das políticas assistenciais públicas de saúde e de suas atribuições é necessário a presença dos diversos atores, que constituem atenção em saúde, nos debates que firmam as estratégias de prevenção e tratamento dos portadores da doença e/ou infectados (VILLARINHO MV, et al., 2013). Do mesmo modo, a epidemia do HIV/AIDS, que nos seus primórdios era tratada como uma questão essencialmente biomédica passou a ser entendida como um evento coletivo e um construto social, que se moldam dentro do contexto de sistemas sociais, culturais, políticos e econômicos interligados no novo conceito de saúde de acordo com a OMS (GUIMARÃES MDC, et al., 2017).

Partindo desses pressupostos, vale ressaltar a grande importância de estudos na linha epidemiológica da tuberculose e do HIV/AIDS para a efetivação de políticas de controle da doença, e em conseguinte, na redução do número de adoecimentos e mortes, considerando que na região norte, existe um elevado número 
de casos do agravo, e um sistema de saúde deficiente com grandes dificuldades de acesso, aliado a situação social. Neste contexto, a Enfermagem vem contribuindo significativamente para a ampliação da noção de cuidado em saúde, tanto do ponto de vista teórico, como da sua práxis, caracterizada pelo entendimento multidimensional envolvendo a questão do HIV/AIDS em todos os seus aspectos (VILLARINHO MV, et al., 2013).

O aumento do número de casos de HIVAIDS, a expansão do acesso e a falência do sistema de saúde, geram dificuldades de acompanhar e monitorar o tratamento, gerando entraves para diagnosticar e tratar os doentes levando maior disseminação da doença (BRASIL, 2016). O conhecimento acerca da doença e do tratamento é importante diante da transmissão. O risco de a pessoa infectar-se com o vírus depende de diversos fatores: intensidade, frequência e duração da exposição à carga viral. Nem todas as pessoas que tem o vírus no organismo desenvolvem a doença. Em pessoas com o sistema imunológico eficiente o vírus acaba aprisionado dentro de uma célula de defesa, o macrófago, podendo permanecer latente por toda a vida, sem manifestar a doença, tal fato ocorre em indivíduos que realizam os cuidados necessários para estagnação da doença (KADRI M e SCHWEICKARDT JC, 2016; CORDEIRO LI, et al., 2017).

O objetivo desse estudo foi descrever as políticas públicas e sociais no contexto de portadores do HIV/Aids.

\section{MÉTODOS}

Tratou-se de um estudo sob método de Revisão Integrativa da Literatura (RIL), com o objetivo de proporcionar novas evidências científicas a partir do tema "Avaliação das Políticas Públicas no contexto de portadores do HIVIAIDS: Uma Revisão Integrativa da Literatura". Como fase inicial formulou-se a seguinte questão: Como está estruturada as Políticas Públicas no contexto de portadores do HIV/Aids? Tal pergunta norteou a busca bibliográfica, filtrando os artigos que possuem coerência com o objeto do estudo.

A busca foi realizada em banco de dados eletrônicos: Medical Literature end Retrieval System on Line (MEDLINE), Literatura Latino-americana e do Caribe em Ciências da Saúde (LILACS), Base de Dados de Enfermagem (BDENF), todos presentes na Biblioteca Virtual da Saúde. Com base na terminologia em saúde DECS, da Biblioteca Virtual em Saúde, foi utilizado na busca de descritores: Políticas Públicas; AIDS; Prevenção. Encontrou-se um total de 189 artigos que após a utilização dos critérios de inclusão e dos critérios de exclusão foram reduzidos a 6 estudos.

As publicações foram organizadas de acordo com suas características, que dizem respeito ao nome do artigo, o ano de publicação, o local de publicação e o objetivo principal. Esses dados foram agrupados em forma de tabela e exibidos nos resultados da pesquisa. Artigos completos foram incluídos na pesquisa, abrangendo os temas e objetivos da pesquisa, relacionados aos temas propostos no projeto de pesquisa entre os anos 2012-2018 redigidos no Brasil e em português. Foram excluídos do estudo artigos que tenham como tema políticas públicas com portadores de outras doenças infecciosas; artigos que necessitem que alguma taxa de pagamento para serem lidos e artigos que relacionem outros temas além de políticas públicas envolvendo HIV/Aids. Este estudo zelou pelos aspectos éticos do Conselho Nacional de Ensino e Pesquisa (CONEP) e, dessa forma, de acordo com as orientações da resolução ํo466 12 de Dezembro de 2012, referente à pesquisa em seres humanos não foi necessária a aprovação da Comissão do Comitê de Ética, em virtude de que a pesquisa será realizada através de levantamento bibliográfico.

\section{RESULTADOS}

Escolheu-se organizar os resultados com base no título do artigo, no estado de publicação, no ano de publicação e na formação do autor (Quadro 1). Respeitou-se todas as descobertas científicas e organizouse em forma de tabela com recursos para melhor observar e compreender cada artigo científico.

Durante a pesquisa foram identificados 6 artigos científicos escritos entre os anos de 2013 a 2015, abordando o tema central das políticas públicas envolvendo o HIV/AIDS. Verifica-se na Tabela 1 que as regiões com maior incidência de pesquisa sobre as políticas públicas envolvendo o HIV/AIDS dentre os anos de 2013 a 2017, a região Sul juntamente com a região Sudeste se prevalecem com 83,33\% do conteúdo de 
pesquisas, já a região Centro-Oeste apresenta 16,67\% dos artigos pesquisados. As regiões Norte e Nordeste apresentaram $0 \%$ de pesquisas, mostrando que na região Norte não há pesquisas publicadas sobre o tema em questão.

Quadro 1 - Características dos artigos científicos que abordam as políticas públicas envolvendo o HIV/AIDS.

\begin{tabular}{|c|c|c|c|c|}
\hline Artigo & Ano & Local & Formação & Título \\
\hline 1 & 2017 & Minas Gerais & Medicina & $\begin{array}{r}\text { Mortalidade por HIV/AIDS no Brasil, 2000-2015: motivos } \\
\text { para preocupação? }\end{array}$ \\
\hline 2 & 2014 & Santa Catarina & Enfermagem & $\begin{array}{c}\text { Percepcaão da AIDS pelos profissionais da saúde que } \\
\text { vivenciaram a epidemia durante o cuidado prestado às } \\
\text { pessoas com a doença, em Florianópolis (SC), Brasil } \\
\text { (1986-2006). }\end{array}$ \\
\hline 3 & 2016 & Rio de Janeiro & Enfermagem & A emergência da AIDS no Amazonas. \\
\hline 5 & 2013 & Santa Catarina & Enfermagem & $\begin{array}{c}\text { Políticas públicas de saúde face à epidemia da AIDS e } \\
\text { a assistência às pessoas com a doença. }\end{array}$ \\
\hline 6 & 2013 & Santa Catarina & Medicina & $\begin{array}{c}\text { Perfil epidemiológico dos pacientes com HIV atendidos } \\
\text { no sul do Estado de Santa Catarina, Brasil, em 2010. }\end{array}$ \\
\hline 2017 & Rio de Janeiro & Enfermagem & Enfermagem e AIDS: saber e paradigma \\
\hline
\end{tabular}

Fonte: Júnior FMA, et al., 2020.

Tabela 1 - Características dos artigos científicos que abordam as políticas públicas envolvendo o HIV/AIDS por região.

\begin{tabular}{ccc}
\hline \multicolumn{3}{c}{ Artigos científicos por regiões no Brasil } \\
\hline Regiões & Pesquisas & $(\%)$ \\
\hline Norte & 0 & $0,00 \%$ \\
Nordeste & 0 & $0,00 \%$ \\
Sul & 3 & $50,00 \%$ \\
Sudeste & 2 & $33,33 \%$ \\
Centro-oeste & 1 & $16,67 \%$ \\
\hline Total & 6 & $100,00 \%$ \\
\hline
\end{tabular}

Fonte: Júnior FMA, et al., 2020.

\section{DISCUSSÃo}

A luta contra o HIV/AIDS não pode ser considerada apenas uma característica assistencial exercida pelos profissionais da saúde, mas principalmente uma ação de criação de políticas públicas sólidas que possam não apenas ofertar um atendimento de qualidade, mas focar na educação em saúde e nas ações preventivas contra doença. É de responsabilidade do estado definir as formas corretas de atuação respeitando a individualidade das pessoas e as responsabilidades assumidas pelos profissionais de saúde, assim seus planos de ações terão maior eficiência e poderão refletir os valores de universalidade, integralidade e equidade previstas pelo Sistema Único de Saúde (SUS) (GUIMARÃES MDC, et al., 2017; SOUSA LRM, et al., 2019).

A dinâmica de criação e amadurecimento das estratégias de prevenção e intervenção ao portador do HIV/AIDS deve ter a participação ativa da sociedade civil e científica, para que juntos todos possam pontuar fragilidades e conquistas dentro dos programas de combate ao HIV. A epidemia doença nos últimos anos vem apresentando uma modificação em seu perfil epidemiológico, isso mostra a dinâmica social que a doença pode apresentar, e assim, é necessário políticas públicas que além possam focar muito além do fator intervencionista, mas que possam avaliar a transcendência da doença (VILLARINHO MV e PADILHA MI, 2014). 
Para Kadri M e Schweickardt JC (2016), por se tratar de uma doença que supera a simples barreira de uma patologia, o fortalecimento das políticas em saúde e o reconhecimento da magnitude da infecção no Brasil e no mundo apresentam inúmeros desafios que instituições públicas e os próprios profissionais da saúde irão enfrentar, e entre os principais desafios que a população busca no combate a doença ainda é a conscientização sobre os atos de risco para contaminação, ou seja, o enfoque no processo de educação em saúde ainda é considerado uma ferramenta determinante no processo de amadurecimento das políticas públicas em saúde ao combate do HIV/AIDS.

Para a consciência saudável de novos padrões de comportamento de prevenção HIV/AIDS as políticas públicas deve envolver diversos setores governamentais que envolvam instituições de educação, de pesquisa básica e avançada e setores econômicos, assim como devem inserir também setores não governamentais que possam trabalhar dentro da perspectiva de ajuda ao portador do HIV/AIDS, como parte terapêutica, de orientação e até de prevenção. Neste contexto, as organizações de movimentos sociais assim com o setores religiosos também se mostram determinantes no processo de evolução do lidar da infecção (VILLARINHO MV, et al., 2013).

O incentivo e fortalecimento das ações por parte dos gestores municipais e estaduais provoca uma profunda transformação positiva na questão social e na área da saúde, principalmente, na perspectiva de melhora da intervenção ao HIV/AIDS. Em um país de dimensões continentais é necessário que cada pequena parte se faça reflexiva em relação a importância do combate a infecção (SCHUELTER-TREVISAL F, et al., 2013 e MONTEIRO RSM, et al., 2019).

Para Xavier LM, et al. (2017), todos os profissionais envolvidos no combate a infecção precisam estar atentos as diversas novas questões que podem surgir no âmbito técnico-científico e até mesmo social da doença. Os avanços e retrocessos que fazem parte do combate ao HIV/AIDS podem representar muito mais do que uma questão política, mas uma nova dinámica adotada pela doença, como novos grupos de risco e variação em sinais e síntomas.

Muitos foram os avanços dos governos estaduais e municipais no Brasil no controle das DSTs, principalmente, no enfrentamento do HIV/AIDS, mas para que as ações possam ter maior prosperidade é necessário fortalecimento do Programa Nacional de HIV/AIDS visando suas diversas áreas de atuação e po amadurecimento das ações, sejam elas no setor primário, secundário e/ou terciário em saúde (GUIMARÃES, MDC et al., 2017 e MOREIRA PA, et al., 2019).

De acordo com Villarinho MV e Padilha MI (2014), com passar dos anos a epidemia da AIDS vem transformando vários pontos de interpretação e refletindo novos desafios para as políticas públicas no país, mas para enfrentar a doença é necessário enfoque não apenas nas políticas públicas, mas em toda a estrutura que sustenta a saúde em nosso país, assim a valorização dos estabelecimentos de saúde, profissionais da saúde, protocolos assistenciais entre outros representam a valorização das ações de prevenção, diagnóstico e até mesmo assistência e cura possível da doença.

Os programas de DST/AIDS não podem ter uma menor representatividade na área da saúde em comparação a outras políticas públicas. Muitas são as difivuldades no setor político que interfere negativamente na prosperidade de alguns programas, como: falta de investimentos tecnológicos e a falta de profissionais capacitados. Assim, é possível observar que não se pode regradir no que já foi alcaçado no cenário de atuação ao combate do HIV/AIDS e sim manter-se em constante evolução e amadurecimento (KADRI M e SCHWEICKARDT JC, 2016 e CORDEIRO LI, et al., 2017).

Mesmo com as significativas conquistas desde a criação do SUS, o processo de construção do sistema único de saúde é contino e precisa cada vez mais quebrar o padrão hospitalocêntrico visando o fortalecimento da atenção básica, assim será possível observar no Brasil a queda no número de mortes, das taxas de incidência e prevalência da doença, e dessa forma, respeitar os princípios e diretrizes que formam o nosso sistema de saúde (VILLARINHO MV, et al., 2013; MONTEIRO SS, et al., 2018).

No cenário de evolução das políticas públicas assistenciais dos portadores de HIV/AIDS é necessário fortalecer também os conceitos de pactuação, ou seja, instituições que visam a valorização da saúde devem, 
preferencialmente, agir conjuntamente pois assim serão amis acertivos nas necessidades biológicas, sociais e mentais. Torna-se muito mais produtivo deixar de pensar apenas na carcterística patológica da deonça, mas em todo o cenário criado pela mesma (SCHUELTER-TREVISAL F, et al., 2013; RIOS LF, et al., 2019).

Para tanto, ações conjuntas com instituições de saúde da mulher, adolescente, criança, saúde mental, vigilância em saúde, saúde da família e saúde comunitária têm sido realizadas para fortalecer o combate ao HIV/AIDS, mas mesmo diante dessa situação, temos obtido sucesso nas políticas públicas relacionadas à epidemia de AIDS no Brasil (XAVIER IM, et al., 2017).

Para realizar o combate às doenças, o Presidente da República promulgou o Plano Nacional de Direitos Humanos (PNDH) em 13 de maio de 1996. No contexto da epidemia de aids, nas duas décadas de epidemia no Brasil, o direito à prevenção e ao tratamento é considerado um direito básico, e os itens específicos do exercício desse direito são garantidos e protegidos, como o direito ao tratamento específico (KADRI M e SCHWEICKARDT JC, 2016).

Responsável por esse conceito, foi criada a Rede de Direitos Humanos e Saúde Mental (HDR) de HIV/AIDS, composta por consultores técnicos das áreas jurídica e da saúde e agências/pessoas mala direta, para garantir que as políticas públicas estejam diretamente vinculadas às doenças sexualmente transmissíveis e $\mathrm{Na}$ área da AIDS, fornecer aconselhamento e promoção sobre direitos humanos e fornecer aconselhamento e promoção para governos locais e organizações não governamentais para garantir vários direitos e combater comportamentos sociopolíticos repetidos de preconceito e discriminação contra pessoas vivendo com HIV / AIDS (SCHUELTER-TREVISAL F, et al., 2013; COSTA MS, et al., 2018).

Atulamente, ainda percebe-se diversas questões de desrespeito aos portadores de HIV/AIDS, dessa forma, observa-se que trabalhar com os conceitos e estigmas das populações são ações valiosas para a evolução do enfrentamento da doença, e assim, permitir maior qualidade de vida para os portadores do vírus (VILLARINHO MV, et al., 2013; GUIMARAES MDC, et al., 2018).

\section{CONSIDERAÇÕES FINAIS}

A prevenção da infecção pelo vírus do HIV por meio de campanhas e políticas públicas em saúde, e o alerta aos agravos a saúde do indivíduo que esta infecção traz consigo, é sem dúvida, uma medida de grande importância para a Saúde Pública e garante a qualidade de vida de parte da população, além de significar melhor qualidade de trabalho para os profissionais envolvidos. Todavia, o conhecimento não deve se restringir apenas ao modo de profilaxia ou aos agravos a saúde que o HIV gera, mas também ao convívio sadio e a quebra de tabus e estereótipos, principalmente os preconceitos, o que dificulta a aceitação por parte da sociedade e assistência por parte de profissionais de saúde, pois o HIV ainda pode ser visto como uma doença específica de um grupo, ou uma consequência de um comportamento sexual inadequado perante a moral. Desde sua descoberta até os dias atuais, a doença ainda encontra-se apoiada em inúmeros problemas políticos assistenciais que diminuíram com o tempo, mas que ainda precisam diminuir mais.

\section{REFERÊNCIAS}

1. BRASIL. Ministério da Saúde. Secretaria de Vigilância em Saúde. Departamento de DST, Aids e Hepatites Virais. Protocolo Clínico e Diretrizes Terapêuticas para Profilaxia Antirretroviral Pós-Exposição de Risco à Infecção pelo HIV. Brasília: Ministério da Saúde, 2016.

2. BRASIL RFG, et al. Avaliação da qualidade de protocolo clínico para atendimento em planejamento familiar de pessoas vivendo com HIV/aids. Rev. esc. enferm. USP, São Paulo, 2018; 52(1): 1-8.

3. CORDEIRO LI, et al. Validação de cartilha educativa para prevenção de HIV/Aids em idosos. Rev. Bras. Enferm., Brasília, 2017; 70(4): 775-782.

4. COSTA MS, et al. Saberes, crenças religiosas e atitudes de mulheres idosas na prevenção ao HIV/Aids. Rev. Bras. Enferm., Brasília, 2018; 71(1): 40-46.

5. GUIMARAES MDC, et al. Mortalidade por HIV/Aids no Brasil, 2000-2015: motivos para preocupação?. Rev. bras. epidemiol., 2017; 20(1): 182-190.

6. KADRI M, SCHWEICKARDT JC. A emergência da AIDS no Amazonas. História, Ciências, Saúde - Manguinhos. 2016. 
7. MONTEIRO RSM, et al. Ações educativas sobre prevenção de HIV/AIDS entre adolescentes em escolas. Enfermería Actual de Costa Rica, San José, 2019; 37(1): 206-222.

8. MONTEIRO SS, et al. Desafios do tratamento como prevenção do HIV no Brasil: uma análise a partir da literatura sobre testagem. Ciência \& Saúde Coletiva [online], 2018; 24(5): 1793-1807.

9. MOREIRA PA, et al. HIV vulnerability among adolescents attending to public schools / Vulnerabilidade ao HIV/AIDS em adolescentes da rede pública de ensino. Revista de Pesquisa: Cuidado é Fundamental Online, 2019; 11(4): 868872.

10. RIOS LF, et al. O drama do sexo desprotegido: estilizações corporais e emoções na gestão de risco para HIV entre homens que fazem sexo com homens. Sex., Salud Soc. (Rio J.), Rio de Janeiro, 2019; 32(1): 65-89.

11. SOUSA LRM, et al. Representações sociais do HIV/Aids por idosos e a interface com a prevenção. Rev. Bras. Enferm., Brasília, 2019; 72(5): 1129-1136.

12. SCHUELTER-TREVISOL F, et al. Perfil epidemiológico dos pacientes com HIV atendidos no sul do Estado de Santa Catarina, Brasil, em 2010. Epidemiol. Serv.Saúde, 2013; 22(1): 87-94.

13. VILLARINHO MV, PADILHA MI. Percepção da AIDS pelos profissionais da saúde que vivenciaram a epidemia durante o cuidado prestado às pessoas com a doença, em Florianópolis (SC), Brasil (1986-2006). Ciênc. saúde coletiva, 2014; 19(6): 1951-1960.

14. VILLARINHO MV, et al. Políticas públicas de saúde face à epidemia da AIDS e a assistência às pessoas com a doença. Rev. bras. enferm., 2013; 66(2): 271-277.

15. XAVIER IM, et al . Enfermagem e AIDS: saber e paradigma. Rev. Latino-Am. Enfermagem, 1997; 5(1): 65-73. 